\title{
General approach to reliable characterization of thin metal films
}

\author{
Tatiana V. Amotchkina, ${ }^{1}$ Vesna Janicki, ${ }^{2}$ Jordi Sancho-Parramon, ${ }^{2}$ \\ Alexander V. Tikhonravov, ${ }^{1}$ Michael K. Trubetskov, ${ }^{1}$ and Hrvoje Zorc ${ }^{2, *}$ \\ ${ }^{1}$ Research Computing Center, Moscow State University, Leninskie Gory, 119992, Moscow, Russia \\ ${ }^{2}$ Rudjer Boskovic Institute, Bijenicka cesta 54, 10002, Zagreb, Croatia \\ ${ }^{*}$ Corresponding author: Hrvoje.Zorc@irb.hr
}

Received 9 November 2010; revised 21 December 2010; accepted 27 December 2010; posted 4 January 2011 (Doc. ID 137919); published 30 March 2011

\begin{abstract}
Optical constants of thin metal films are strongly dependent on deposition conditions, growth mode, and thickness. We propose a universal characterization approach that allows reliable determination of thin metal film optical constants as functions of wavelength and thickness. We apply this approach to determination of refractive index dispersion of silver island films embedded between silica layers. (C) 2011 Optical Society of America
\end{abstract}

OCIS codes: $\quad 310.3840,310.6860$.

\section{Introduction}

In the last 20 years, the interest in research of thin metal films and metal-dielectric coatings has increased significantly [1-16]. Thin metal films possess a potential in design and manufacture of multilayer structures with sophisticated spectral performances over wide wavelength ranges. It was demonstrated in [17] that there are design problems that cannot be solved without using thin metal layers. In [18], it was shown that the multilayer coatings with thin metal layers can be competitive with pure dielectric coatings, even in applications where using metal layers is not absolutely necessary.

Depending on the thickness of the layer and deposition conditions, metal can grow as compact film or as metal island film (MIF). In this study, the term thin metal films refers to both mentioned types of metal films.

To design and produce high quality metaldielectric optical coatings with desired spectral characteristics, it is required to know the optical constants of thin metal films with high accuracy. As opposed to bulk metal films, whose refractive indices

0003-6935/11/101453-12\$15.00/0

(C) 2011 Optical Society of America and extinction coefficients can be found in literature [19-21], in the modern state of art, there are no commonly used databases of optical constants of thin metal films. The reason is that optical constants of thin metal films are strongly dependent on film thickness. This is due to electron confinement effects that usually result in higher absorption losses in comparison with bulk materials $[15,22]$.

Depending on fabrication conditions and due to the poor wetting of some metals on dielectric surfaces, deposition of a few nanometers of metal often results in island films, i.e., films composed by clusters of nanometric dimensions [1]. The optical properties of such films are completely different than those of pure metals due to the phenomenon of surface plasmon resonance of free electrons in metal clusters, particularly in the vicinity of plasmon resonance frequency $[1,3,8,12,14,23]$. These unique optical properties enable multiple applications of MIFs, which can be incorporated into coatings for combined optoelectronic and nonlinear devices, decorative coatings, spectrally selective absorbers, biosensors, or forgery-proof coatings $[2-4,15,16,24,25]$. The surface plasmon resonance is strongly dependent on the cluster shape and size and, therefore, the optical behavior of MIFs is highly dependent on the amount of 
deposited metal [1,11-14,23]. The dielectric environment strongly influences the optical properties of these films as well $[6,7]$. Although MIFs are not compact layers, if the size of clusters is small compared to the wavelength of light, they can be treated as layers with effective optical constants and thicknesses. These layers actually correspond to a composite of metal clusters and their dielectric environment (metal-dielectric composites).

Characterization results related to different types of thin metal films can be found in many publications (see, for example, $[5,12-14,16,26-28])$. In these publications, different sets of experimental data were used and various characterization approaches were applied. Characterization results were presented as wavelength dependencies of refractive indices and extinction coefficients of thin metal films. Evidently, it is not realistic to obtain optical parameters of thin metal films for each particular set of deposition parameters and deposition conditions. At the same time, it is reasonable to elaborate a general characterization approach that would allow one to easily obtain wavelength and thickness dependencies of optical constants for any type of thin metal films.

It should be noted here that characterization of thin metal films is not a straightforward task. The key problem of simultaneous extraction of refractive index, extinction coefficient, and film thickness of very thin absorbing films from optical measurements has been previously discussed [26,27]. In addition to this, the application of complicated multiparametric models for optical constants of thin metal films can easily lead to instable determination of model parameters and makes it impossible to obtain thickness dependencies of optical constants.

In the present paper, we elaborate a general characterization approach, including experimental procedures and mathematical apparatus, for reliable determination of wavelength and thickness dependencies of optical constants of thin metal films. We demonstrate application of this new approach in characterization of silver island films. In Section 2 , we propose a set of experimental samples that can be used for determination of wavelength and thickness dependencies of optical constants of thin metaldielectric composite films. In this section, we describe our samples of MIFs and the measurement data that we have at our disposal. In Section 3, we present a model of a thin metal film and provide theoretical considerations and results of numerical simulation to find which combination of measurement data sets allows one to obtain reliable optical parameters of thin metal films. In Section 4, we describe our characterization procedure in detail and provide characterization results related to silver island films. In this section, we derive wavelength and thickness dependencies of refractive indices and extinction coefficients of silver island films. Our conclusions are given in Section $\underline{5}$.
In fact, in this paper, we propose an approach for reliable characterization of not one, but of a set of metal film samples differing in thickness. Our approach covers a whole chain, starting from production of a test sample to determination of the dependencies of optical constants of thin metal films on two parameters: wavelength and thickness. These dependencies can be easily incorporated to algorithms for design of multilayer optical coatings. The results of this paper will be useful for optical coating designers and researchers working with thin compact metal films and metal-dielectric composite films.

\section{Experimental Samples and Measurement Data}

To provide reliable determination of refractive indices and extinction coefficients as functions of film thickness, we propose to produce a set of test samples with increasing amounts of deposited metal. Deposition conditions and parameters must be the same for all test samples.

In the course of our research, a set of eight test samples of silver films embedded between silica layers on BK7 substrates were produced. We shall refer to these samples as $\mathrm{S} 1, \ldots, \mathrm{S} 8$. The samples were deposited by electron beam evaporation. Substrates were preheated to $220^{\circ} \mathrm{C}$ to enhance island growth of metal $[1,29]$. The base pressure was $5 \cdot 10^{-6}$ Torr. Deposition rates of $\mathrm{SiO}_{2}$ and $\mathrm{Ag}$ were $1 \mathrm{~nm} \mathrm{~s}^{-1}$ and $0.1 \mathrm{~nm} \mathrm{~s}^{-1}$, respectively. The mass thickness of deposited metal was controlled by quartz crystal monitoring.

The mass thicknesses of deposited silver are presented in the second column of Table $1 . \mathrm{SiO}_{2}$ layers are the same in all test samples and their thicknesses were equal to $d=78 \mathrm{~nm}$.

It was verified earlier by nonoptical methods that, under these deposition conditions, silver forms not compact, but island, films [29-31]. The reason to surround $\mathrm{Ag}$ with $\mathrm{SiO}_{2}$ was to provide the same embedding media to all clusters, as the optical properties of MIFs depend strongly on the dielectric environment $[7,23]$. In addition to this, the $\mathrm{SiO}_{2}$ outermost layers preserve Ag clusters to be exposed directly to the influence of atmosphere.

To obtain reliable characterization results, it is important to choose an appropriate combination of measurement data. We tested which combination of measurement data provides a unique and stable

Table 1. Mass and Effective Thicknesses of Ag-SiO ${ }_{2}$ Composite Films

\begin{tabular}{lcc}
\hline Sample & Mass Thickness $(\mathrm{nm})$ & Effective Thickness $\delta(\mathrm{nm})$ \\
\hline S1 & 3.0 & 6.0 \\
S2 & 6.0 & 12.0 \\
S3 & 9.0 & 18.0 \\
S4 & 11.0 & 19.9 \\
S5 & 12.0 & 23.0 \\
S6 & 13.5 & 24.6 \\
S7 & 15.0 & 26.8 \\
S8 & 18.0 & 29.5 \\
\hline
\end{tabular}


solution of the characterization problem. We took into account, also, that, from a mathematical point of view, characterization problems can be considered as inverse problems. A larger amount of measurement data delivers more information and reduces uncertainty in optical parameter determination $[32,33]$.

At our disposal, we had spectral photometric and ellipsometric data. Reflectance $(R)$ and transmittance $(T)$ measurements of samples were taken at near-normal incidence in the spectral range from 350 to $1100 \mathrm{~nm}$ using a PerkinElmer Lambda 25 spectrometer. Ellipsometric angles $\Psi$ and $\Delta$ for three angles of incidence of $45^{\circ}, 55^{\circ}$, and $65^{\circ}$ in the spectral range from 285 to $2200 \mathrm{~nm}$ were taken using a Woollam VASE variable angle spectroscopic ellipsometer.

\section{Theoretical Study and Numerical Simulation on the Reliability of Optical Parameter Determination}

\section{A. Modeling of Thin Metal Films and Describing Optical Constant Dispersion Behavior}

In the case of thin compact metal films, special modeling is not required. These films can be considered in the frame of the classical approach. This is not the case for MIFs. Schematic representation of an MIF embedded between two dielectric layers is shown in the left part of Fig. 1. Such films have complicated morphology; the sizes and shapes of particles are different. Results of rigorous modeling of such films can be found in $[4,34,35]$. However, in the present research, we concentrate not on the structure of MIFs, but on determination of the optical constants of such films. It is important for our research that, because the sizes of metal particles and distances between them are small compared to the wavelength, the film can be treated as optically homogeneous [36]. We consider an MIF embedded into a silica matrix as a layer of effective thickness $\delta$. Because of the island structure of the film, the effective thickness of the film does not coincide with the mass thickness of the film.

The model sketched in Fig. 1 is used to find out the effective optical constants of the $\mathrm{SiO}_{2}-\mathrm{Ag}$ composite. Regarding the $\mathrm{SiO}_{2}$ layers, their optical constants were previously determined by optical measurements on $\mathrm{SiO}_{2}$ single layers deposited under the same conditions. The obtained optical constants are close to usual literature values [19]. In the spectral range of interest, the effective optical behavior of the MIFs is characterized by the collective oscillations of electrons in metal islands, known as surface plasmons. Ideally, the absorption related to the surface plasmon resonance of an isolated small spherical metal particle presents a Lorentzian line shape [23]. However, due to the presence of shape and size distributions of the metal islands and the electromagnetic coupling among particles, the surface plasmon resonance becomes inhomogeneously broadened. Thus, it is not straightforward to choose a versatile and simple expression that describes the dispersion of the optical constants of the metaldielectric composite.

Various models can be used for representing refractive indices and extinction coefficients of composite materials. These models are based on effective medium theories and assume that composite films are formed as mixtures of materials that are present as such phases of the microstructure that the medium can be macroscopically treated as homogeneous [36]. Model parameters in the case of application of these formulas are filling material fraction and depolarization [36]. However, due to the complicated morphology of films with metal inclusions, the models based on effective medium theories cannot be applied for representing the optical constants of such films. The corresponding discussion can be found in [36]. In our approach, we represent an MIF as an optically homogeneous layer with refractive index $n(\lambda)$ and extinction coefficient $k(\lambda)$ (see Fig. 1).

In the frame of the most commonly used characterization techniques, dispersion of materials are described with appropriate models. The model parameters are usually obtained basing on the minimization of the discrepancy function, estimating closeness between model and experimental spectral characteristics. In the case of metal films, this approach may lead to unstable characterization results for the following reason. It is known from a number of publications (see, for example, $[5,8,11,13,14,19$ $21,36])$ that wavelength dependencies of the optical constants of bulk metals and thin metal films in the visible and infrared spectral ranges are quite

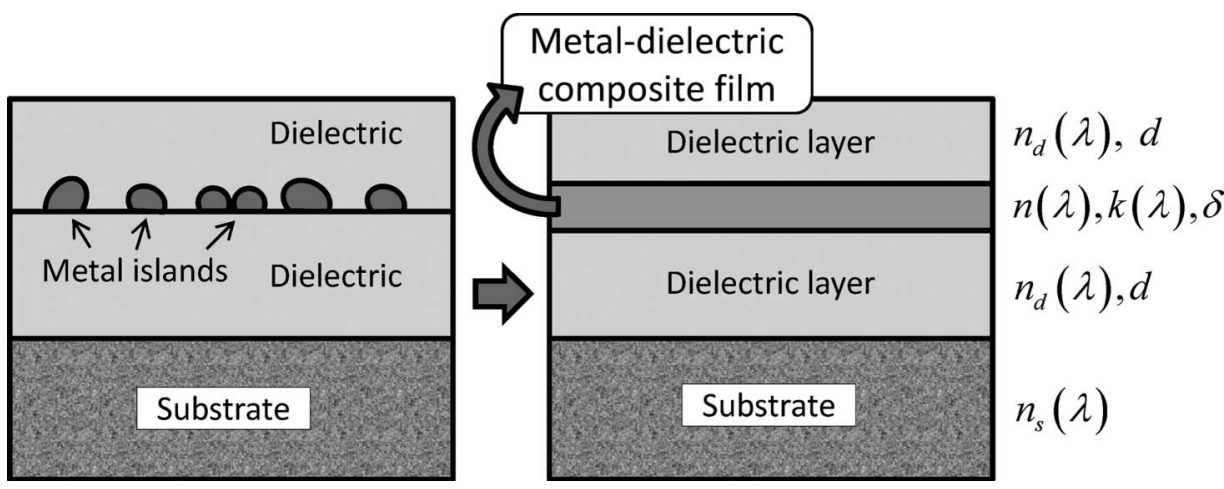

Fig. 1. Modeling of MIF. 
complicated and cannot be described by simple parametric models. By using multiparametric models, it is possible to provide an excellent fitting of measurement data but it is not guaranteed that the obtained parameters have physical sense. In addition to this, instabilities of calculated parameters cause difficulties in obtaining reliable dependencies of optical constants on film thickness.

In our approach, we propose using nonparametric models for description of $n$ and $k$ wavelength dependencies. We assume that $n(\lambda)$ and $k(\lambda)$ are arbitrary smooth functions. The main difference of this approach in comparison with a single-wavelength characterization technique is that we take into account demand on the smoothness of $n$ and $k$ wavelength dependencies in the course of characterization. In [37], the approach assuming smoothness of $n(\lambda)$ and $\overline{k(\lambda)}$ functions was applied to the characterization of quasi-rugate filters. A nonparametric model with additional demand on $n$ and $k$ smoothness was used for thin film characterization in [38] and further developed in $[26,27,39,40]$. In the case of the nonparametric model, the discrepancy function is written in the following way $[26,27]$ :

$$
\begin{aligned}
\mathrm{DF}= & \sum_{j=1}^{L}\left[\frac{S\left(n\left(\lambda_{j}\right), k\left(\lambda_{j}\right), \delta, \lambda_{j}\right)-\hat{S}\left(\lambda_{j}\right)}{\Delta S_{j}}\right]^{2} \\
& +\alpha_{1} \sum_{j=1}^{L}\left[n^{\prime \prime}\left(\lambda_{j}\right)\right]^{2}+\alpha_{2} \sum_{j=1}^{L}\left[k^{\prime \prime}\left(\lambda_{j}\right)\right]^{2},
\end{aligned}
$$

where $S(n, k, \delta, \lambda)$ is the theoretical spectral characteristic of the sample, $\hat{S}(\lambda)$ is the measured spectral characteristic, $\Delta S_{j}$ is tolerances, and $\left(\lambda_{j}\right), j=1, \ldots L$, is the wavelength grid in the spectral range of interest $\left[\lambda_{d} ; \lambda_{u}\right]$. In Eq. (1), $n^{\prime \prime}(\lambda)$ and $k^{\prime \prime}(\lambda)$ denote numerical second-order derivatives of refractive index and extinction coefficient. The second and the third terms in Eq. (1) specify the additional demands on smoothness of $n$ and $k$ wavelength dependencies. introducing penalties for nonsmooth behavior of these functions. Introduction of the second derivatives to Eq. (1) also permits us to exclude linear variation terms in the $n(\lambda)$ and $k(\lambda)$ wavelength dependencies from the penalty additions in Eq. (1). The parameters $\alpha_{1}$ and $\alpha_{2}$ in Eq. (1) are weight factors controlling the correlation between the smoothness demand and demand on good fit of measurement data by theoretical data. A characterization algorithm based on minimization of the discrepancy function in Eq. (1) is incorporated to the OptiRE module of OptiLayer thin film software [39-41]. We use this algorithm in our characterization process.

\section{B. Uncertainty in Determination of Optical Parameters from Spectral Photometric Data}

In [26], an important conclusion about the reliability of characterization results related to thin metal films was done. In this work, reflectance and transmittance of single thin metal layers and two-layer metal-dielectric coatings were considered in the frame of first-order perturbation theory. It was shown that, for small values of thickness $\delta$, approximate reflectance and transmittance coefficients do not depend on separate values $n, k$, and $\delta$, but on the combinations of parameters $2 n k \delta=\operatorname{Im} \epsilon \delta$ and $\left[n^{2}-k^{2}\right] \delta=\operatorname{Re} \epsilon \delta$, where $\epsilon$ is the effective dielectric function. This means it is not possible to separately determine $n, k$, and $\delta$ values from reflectance and transmittance measurements only.

In the present work, we applied the first-order perturbation theory to derive approximate expressions for $R$ and $T$ of a symmetrical three-layer structure sketched in the right part of Fig. 1. For small values of $\delta$ we obtained

$$
\begin{aligned}
R(\lambda) \approx & R_{1}+\frac{2 \pi}{\lambda}\left(A_{R} \cdot 2 n(\lambda) k(\lambda)+B_{R} \cdot\left[n^{2}(\lambda)\right.\right. \\
& \left.\left.-k^{2}(\lambda)\right]+C_{R}\right) \delta, \\
T(\lambda) \approx & T_{1}+\frac{2 \pi}{\lambda}\left(A_{T} \cdot 2 n(\lambda) k(\lambda)+B_{T} \cdot\left[n^{2}(\lambda)\right.\right. \\
& \left.\left.-k^{2}(\lambda)\right]+C_{T}\right) \delta,
\end{aligned}
$$

where $R_{1}=R_{1}(\lambda)$ and $T_{1}=T_{1}(\lambda)$ are reflectance and transmittance coefficients of a single dielectric layer with refractive index $n_{d}=n_{d}(\lambda)$ and thickness $2 d$. The parameters $A_{R, T}, B_{R, T}$, and $C_{R, T}$ in Eq. (2) are dependent on the parameters $n_{d}, n_{s}$, and $d$ and on the wavelength $\lambda$. We do not present these dependencies here because they are too cumbersome. An interested reader can find the expressions for $A_{R, T}, B_{R, T}$, and $C_{R, T}$ in Appendix A.

We checked numerically that, for a wide range of $n$ and $k$ values, it is valid that $\left|C_{R, T}\right| \ll\left|A_{R, T} \operatorname{Im} \epsilon\right|$ and $\left|C_{R, T}\right| \ll\left|B_{R, T} \operatorname{Re} \epsilon\right|$. Hence, we can approximate $R$ and $T$ in the following way:

$$
\begin{aligned}
& R(\lambda) \approx R_{1}+\frac{2 \pi}{\lambda}\left(A_{R} \cdot 2 n(\lambda) k(\lambda)+B_{R} \cdot\left[n^{2}(\lambda)-k^{2}(\lambda)\right]\right) \delta, \\
& T(\lambda) \approx T_{1}+\frac{2 \pi}{\lambda}\left(A_{T} \cdot 2 n(\lambda) k(\lambda)+B_{T} \cdot\left[n^{2}(\lambda)-k^{2}(\lambda)\right]\right) \delta .
\end{aligned}
$$

It follows from Eq. (3) that, similar to the case considered in $[26,27]$, reflectance and transmittance of a three-layer metal-dielectric coating depend on the combinations of parameters $2 n(\lambda) k(\lambda) \delta=\operatorname{Im} \epsilon \delta$ and $\left[n^{2}(\lambda)-k^{2}(\lambda)\right] \delta=\operatorname{Re} \epsilon \delta$. Dependence of $R$ and $T$ on the combinations but not on the separate values of $n, k$, and $\delta$ causes uncertainty in determination of the optical constants and thickness of a thin metal film from photometric data.

For the sake of clearness, we illustrate this uncertainty using a characterization example. Consider measurement reflectance and transmittance data of sample S2. These data are shown in Fig. $\underline{2}$ by crosses. We searched for $n$ and $k$ wavelength dependencies based on the minimization of the discrepancy function in Eq. (1). In the course of minimization, we 

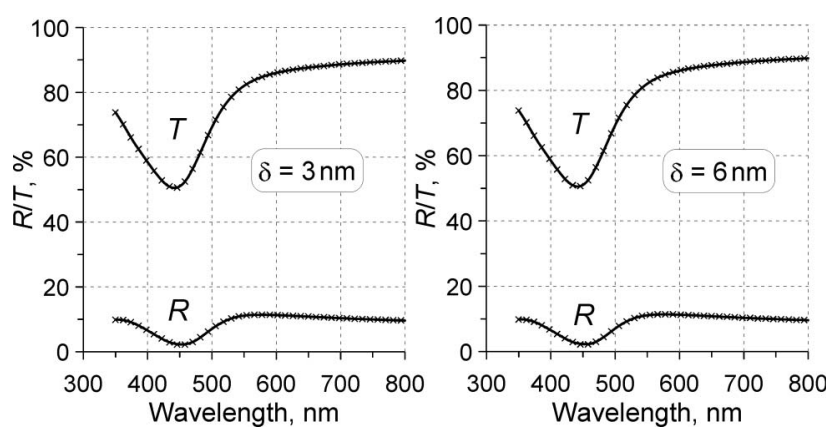

Fig. 2. Fitting of measurement reflectance and transmittance data by theoretical spectral characteristics obtained in the course of two characterization attempts (see Subsection 3.B for details).

fixed the value of thickness $\delta$. We made two characterization attempts: with $\delta_{1}=3 \mathrm{~nm}$ and $\delta_{2}=6 \mathrm{~nm}$. We knew a priori that both values of $\delta$ are not correct because the effective thickness of the metaldielectric composite film in this sample is definitely higher than the mass thickness of the film, which is equal to $6 \mathrm{~nm}$. However, in both characterization attempts, we obtained excellent fittings of measurement data (see Fig. 2). The obtained wavelength dependencies for both cases $n^{(1)}(\lambda), n^{(2)}(\lambda)$, and $k^{(1)}(\lambda)$, $k^{(2)}(\lambda)$ are shown in Fig. 3 . We checked that ratios of $n$ and $k$ values from two attempts are very close: $n^{(1)}(\lambda) / n^{(2)}(\lambda) \approx k^{(1)}(\lambda) / k^{(2)}(\lambda) \approx 1.4 \approx \sqrt{2}$. Therefore, we obtained two completely different sets of optical parameters of this metal-dielectric composite film. These parameters, however, are connected by the relations: $\delta_{2}=2 \delta_{1}, n^{(2)} \approx n^{(1)} / \sqrt{2}, k^{(2)} \approx k^{(1)} / \sqrt{2}$. These relations are in a full agreement with the approximate formulas in Eq. (3): despite the values of $n^{(1)}, k^{(1)}, \delta_{1}$, and $n^{(2)}, k^{(2)}$ and $\delta_{2}$ are different; the combinations $2 n(\lambda) k(\lambda) \delta=\operatorname{Im} \epsilon \delta$ and $\left[n^{2}(\lambda)-\right.$ $\left.k^{2}(\lambda)\right] \delta=\operatorname{Re} \epsilon \delta$ take the same values.

Evidently, the relations of Eq. (3) provide accurate approximations of reflectance and transmittance coefficients for a limited range of parameters $n, k$, and $\delta$. In the course of our research, we compared values of $R$ and $T$ calculated using exact and approximate formulas for various values of parameters $n, k$, and $\delta$. The results of this comparison showed that, in
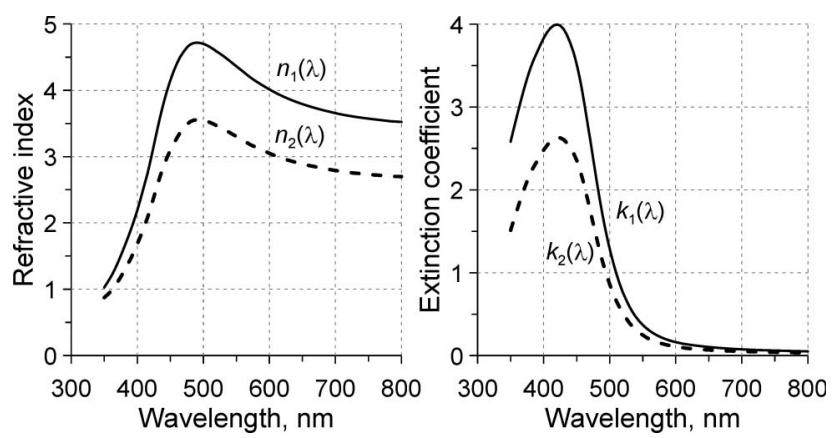

Fig. 3. Comparison of the refractive indices and extinction coefficients calculated in the course of the first (solid curves) and the second (dashed curves) characterization attempts (see Subsection 3.B for details). the case when $0.5 \leq n, k \leq 3$, the relations in Eq. (3) provide good approximations up to $\delta$ values of $5-6 \mathrm{~nm}$ and, in the case when $0.1 \leq n, k \leq 0.5$ or $3 \leq n, k \leq 5$, the relations in Eq. (3) approximate $R$ and $T$ up to $\delta$ equal to $2-3 \mathrm{~nm}$. Typically, the refractive index and extinction coefficients of metal films vary significantly with respect to the wavelength and take values from 0.1 to 5 . This allows us to state that, in the case of very thin metal films of thicknesses up to $5 \mathrm{~nm}$, the relations in Eq. (3) provide accurate approximations of reflectance and transmittance coefficients and, therefore, prove the instability of optical parameter determination from $R$ and $T$ data.

Depending on deposition conditions, metaldielectric composite films of thicknesses of two-three dozens of nanometers can be still not compact and possess optical properties mainly dominated by the surface plasmon resonance of metal clusters. It is necessary to obtain reliable characterization results for metal-dielectric composite films with thicknesses of up to $20-30 \mathrm{~nm}$. In this case, the formulas in Eq. (3) obtained in the frame of first-order perturbation theory no longer provide good approximations of $R$ and $T$ values. In spite of this, there is still uncertainty in optical parameter determination from reflectance and transmittance measurements. To demonstrate the presence of uncertainty in determination of $n, k$, and $\delta$ values from $R$ and $T$ data, we performed numerical simulation. In the course of this simulation, we modeled two designs and compared theoretical reflectance data of these designs and transmittance data of these two designs. The structure of those designs is shown in the right side of Fig. 1. We specified parameters of metal-dielectric composite films $n^{(1)}, k^{(1)}, \delta_{1}$, and $n^{(2)}, k^{(2)}, \delta_{2}$ in these two modeled designs so that the following relations are satisfied: $n^{(2)}(\lambda)=c n^{(1)}(\lambda), k^{(2)}(\lambda)=c k^{(1)}(\lambda)$, and $\delta_{2}=\delta_{1} / c^{2}$, where $c$ is a parameter. In this case, the combinations of parameters $2 n(\lambda) k(\lambda) \delta=\operatorname{Im} \epsilon \delta$ and $\left[n^{2}(\lambda)-k^{2}(\lambda)\right] \delta=\operatorname{Re} \epsilon \delta$ take the same values. In our numerical simulations, we varied the value of parameter $c$ from 0.95 to 1.05 , which correspond to the variations of $n$ and $k$ from $-5 \%$ to $5 \%$ and variations of $\delta$ from $-10 \%$ to $10 \%$. For each $c$ value, we calculated the standard deviations $\sigma_{R}, \sigma_{T}$ between reflectance and transmittance corresponding to two modeled designs. In Fig. 4, we present an example of such a comparison. The solid curves in Fig. 4 show reflectance and transmittance of a modeled design containing a metal-dielectric composite film with refractive index $n^{(1)}(\lambda)$ and extinction coefficient $k^{(1)}(\lambda)$ corresponding to sample S4 and with thickness $\delta_{1}=20 \mathrm{~nm}$. Curves with markers show reflectance and transmittance coefficients of a model design containing a metal-dielectric composite film with refractive indices $n^{(2)}(\lambda)=c n^{(1)}(\lambda)$ and extinction coefficients $k^{(2)}=c k^{(1)}(\lambda)$, where $c=0.95$ and 1.05, respectively. Deviations $\sigma_{R}$ and $\sigma_{T}$ are about $1.2 \%$. This result and many other results of numerical simulations for various values of $n, k, \delta$, and $c$ show that the standard deviations $\sigma_{R}$ and $\sigma_{T}$ do not exceed 

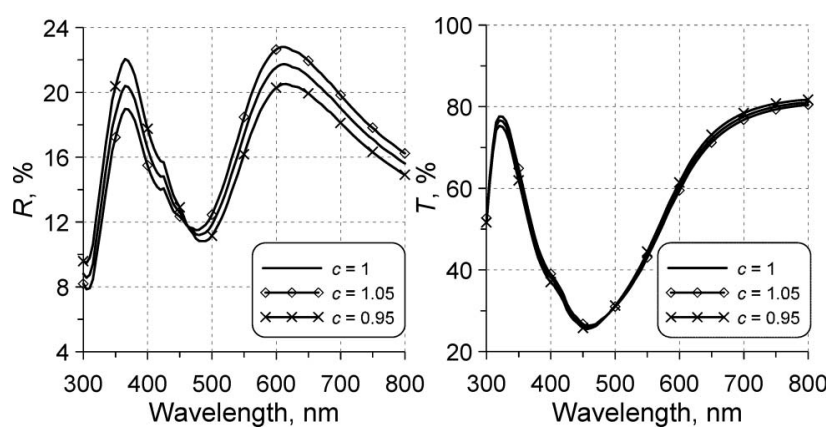

Fig. 4. Comparison of reflectance and transmittance of model designs corresponding to different values of parameter $c$ (see Subsection 3.B for details).

$1 \%-1.5 \%$; that is comparable with measurement accuracy. At the same time, the 5\% errors in optical constants and $10 \%$ errors in thickness of thin metaldielectric composite film are quite significant levels of error. Our numerical results demonstrate that the parameters $n, k$, and $\delta$ obtained in the course of characterization process based only on $R$ and $T$ measurements may differ from the true ones significantly. In the case when one has only transmittance and reflectance measurement data, additional information is required. This information can be provided, for example, by measurement of film thickness using other experimental tools.

\section{Stability of Determination of Thin Metal Film Optical Parameters from Ellipsometric Measurements}

It is known that ellipsometric angles $\Psi$ and $\Delta$ are sensitive to even very thin surface overlayers of dielectric films (see, for example, [39,40]). In this study, we prove analytically that ellipsometric angles $\Psi$ and $\Delta$ are sensitive to thin metal layers embedded between dielectric layers. For this purpose, we applied the first-order perturbation theory to derive approximate expressions of ellipsometric angles $\Psi$ and $\Delta$. We assumed that the thickness of thin metal layer $\delta$ is a small parameter and obtained the following approximate expressions:

$$
\begin{aligned}
\Psi(\lambda) \approx & \Psi_{1}+\frac{2 \pi}{\lambda}\left(A_{\Psi} \cdot 2 n(\lambda) k(\lambda)+B_{\Psi}\right. \\
& \left.\cdot\left[n^{2}(\lambda)-k^{2}(\lambda)\right]+C_{\Psi}\right) \delta, \\
\Delta(\lambda) \approx & \Delta_{1}+\frac{2 \pi}{\lambda}\left(A_{\Delta} \cdot 2 n(\lambda) k(\lambda)+B_{\Delta}\right. \\
\cdot & {\left.\left[n^{2}(\lambda)-k^{2}(\lambda)\right]+C_{\Delta}\right) \delta . }
\end{aligned}
$$

In Eq. (4) $), A_{\Psi, \Delta}, B_{\Psi, \Delta}$, and $C_{\Psi, \Delta}$ are parameters dependent on $n_{d}, d, n_{s}, \lambda$, and incidence angle $\theta ; \Psi_{1}=\Psi_{1}(\lambda)$ and $\Delta_{1}=\Delta_{1}(\lambda)$ are ellipsometric angles of a single layer with refractive index $n_{d}$ and physical thickness $2 d$. In the general case, the expressions for parameters in Eq. (4) are too cumbersome and we present them in Appendix A. In the special case when the refractive index of dielectric layers $n_{d}$ is close to the refractive index of the substrate $n_{s}$, the expression for $\Delta$ can be written in a compact form:

$$
\begin{aligned}
\Delta(\lambda) & \approx \frac{4 \pi}{\lambda}[A \sin (2 \phi) \xi-A \cos (2 \phi) \eta+C \cos (2 \phi)] \delta, \\
A & =\frac{q_{a}^{p}}{\left(q_{a}^{p}\right)^{2}-\left(q_{s}^{p}\right)^{2}}-\frac{q_{a}^{s}}{\left(q_{a}^{s}\right)^{2}-\left(q_{s}^{s}\right)^{2}}, \\
C & =\frac{q_{a}^{p}\left(q_{s}^{p}\right)^{2}}{\left(q_{a}^{p}\right)^{2}-\left(q_{s}^{p}\right)^{2}}-\frac{q_{a}^{s}\left[\left(q_{s}^{s}\right)^{2}+\sin ^{2} \theta\right]}{\left(q_{a}^{s}\right)^{2}-\left(q_{s}^{s}\right)^{2}},
\end{aligned}
$$

where $\phi=2 \pi / \lambda\left(n_{d}^{2}-\sin ^{2} \theta\right)^{1 / 2} d$, and $q_{s, a}^{p, s}$ are the effective refractive indices of the substrate and ambient medium in the case of $p$ - and $s$-polarization.

In the special case of a single thin metal layer on the substrate, Eq. (ㄴ) can be written in a simple way:

$$
\begin{aligned}
\Psi(\lambda) \approx & \Psi_{0} \pm \frac{2 \pi}{\lambda} \frac{q_{a}^{p}\left[\left(q_{s}^{s}\right)^{2}-\left(q_{a}^{s}\right)^{2}\right]-q_{a}^{s}\left[\left(q_{s}^{p}\right)^{2}-\left(q_{a}^{p}\right)^{2}\right]}{\left(q_{s}^{s} q_{a}^{p}-q_{a}^{s} q_{s}^{p}\right)^{2}+\left(q_{a}^{s} q_{a}^{p}-q_{s}^{s} q_{s}^{p}\right)^{2}} \\
& \cdot 2 n(\lambda) k(\lambda) \delta, \\
\Delta(\lambda) \approx & -\frac{4 \pi}{\lambda}\left[A \cdot\left[n^{2}(\lambda)-k^{2}(\lambda)\right]-C\right] \delta, \\
\Psi_{0}= & \arctan \left[\frac{\left|q_{a}^{p}-q_{s}^{p}\right|}{q_{a}^{p}+q_{s}^{p}} \cdot \frac{q_{a}^{s}+q_{s}^{s}}{q_{s}^{s}-q_{a}^{s}}\right] .
\end{aligned}
$$

The signs "+" and "-" in Eq. (6) correspond to the cases when incidence angle is higher or lower than Brewster's angle.

It is seen from Eqs. (4) and (5) that the ellipsometric angles are dependent on combinations of parameters $2 n k \delta=\operatorname{Im} \epsilon \delta,\left[n^{2}-k^{2}\right] \delta=\operatorname{Re} \epsilon \delta$ and separately on film thickness $\delta$. To investigate the sensitivity of $\Psi$ and $\Delta$ angles to $\delta$, we compared numerically the values $A_{\Psi, \Delta} \operatorname{Im} \epsilon$ and $B_{\Psi, \Delta}$ Re $\epsilon$ with the values $C_{\Psi, \Delta}$. We performed this comparison for various values of $n$ and $k$ for typical values of $n_{d}$, as well as for various values of $d$. First, we specified $n$ and $k$ as constant values from the range $[0.1 ; 5]$. Then we specified wavelength dependencies for $n(\lambda)$ and $k(\lambda)$ corresponding to optical constants of bulk gold, silver, copper, nickel, and chromium $[19,20]$. In the course of the comparison, we found o that the values of $A_{\Psi, \Delta} \operatorname{Im} \epsilon, B_{\Psi, \Delta} \operatorname{Re} \epsilon$, and $C_{\Psi, \Delta}$ are comparable in magnitude in the visible spectral range. In the case of a single layer, the parameters $A \operatorname{Re} \epsilon$ and $C$ in Eq. (6) are also comparable in magnitude. Basing on the approximate formulas in Eqs. (4)-(6) and on the results of numerical comparison described above, we conclude that ellipsometric data allow separate determination of optical parameters $n, k$, and $\delta$. Because of this conclusion, we propose to perform characterization of test samples based on ellipsometric data. Reflectance and transmittance data can be useful for verification of characterization results.

\section{Characterization of Test Samples}

\section{A. Characterization Process and Verification of Results}

In this subsection, we describe our characterization process in detail and present characterization results obtained for test samples of silver island films described in Section 2. According to the conclusion 
made in the previous section, in our characterization process, we used ellipsometric data. In the course of the characterization process, we searched for wavelength dependencies of optical constants of $\mathrm{Ag}$ $\mathrm{SiO}_{2}$ composite films $n(\lambda), k(\lambda)$. We also searched for thicknesses of dielectric layers surrounding $\mathrm{Ag}-\mathrm{SiO}_{2}$ composite film. Because $\mathrm{SiO}_{2}$ of the cover later also fills the space between islands, it is not expected that the effective thickness of this layer is equal to that of the first layer. In this case, the discrepancy function in Eq. (1) can be written in the following way: spond to the higher values of mass thicknesses. The obtained optical constants are characterized by a strong resonance in the visible range, which can be associated with the surface plasmon of metal clusters. This resonance increases in intensity as the amount of deposited metal increases; that can be explained by a higher filling fraction of silver in the metal-dielectric composite $[23,42]$. The shift to higher wavelengths can be related also to the higher metal filling fraction and to the fact that larger clusters usually present a more elliptical shape than smaller clusters [43]. Additionally, a small absorp-

$$
\begin{aligned}
\mathrm{DF}= & \sum_{K=1}^{3} \sum_{j=1}^{190}\left[\frac{\Psi\left(n\left(\lambda_{j}\right), k\left(\lambda_{j}\right), \delta, \lambda_{j}, d^{(1)}, d^{(2)}, \theta_{K}\right)-\hat{\Psi}\left(\lambda_{j}, \theta_{K}\right)}{\Delta \Psi_{j}}\right]^{2}+\sum_{K=1}^{3} \sum_{j=1}^{190}\left[\frac{\Delta\left(n\left(\lambda_{j}\right), k\left(\lambda_{j}\right), \delta, \lambda_{j}, d^{(1)}, d^{(2)}, \theta_{K}\right)-\hat{\Delta}\left(\lambda_{j}, \theta_{K}\right)}{\Delta \Delta_{j}}\right]^{2} \\
& +\alpha_{1} \sum_{j=1}^{190}\left[n^{\prime \prime}\left(\lambda_{j}\right)\right]^{2}+\alpha_{2} \sum_{j=1}^{190}\left[k^{\prime \prime}\left(\lambda_{j}\right)\right]^{2}
\end{aligned}
$$

where $\theta_{1}=45^{\circ}, \theta_{2}=55^{\circ}$, and $\theta_{3}=65^{\circ} ;\left(\lambda_{j}\right)$ is the wavelength grid in the spectral range from 285 to $2200 \mathrm{~nm}$; and $d^{(1)}$ and $d^{(2)}$ are the thicknesses of surrounding dielectric layers. In the course of the characterization process, the values of $d^{(1)}$ and $d^{(2)}$ were varied from $0.9 d$ to $1.1 d$. The values of $\delta$ were varied in a wide range between the value that is twice less and the value that is three times greater than mass thickness. The parameters $\alpha_{1}$ and $\alpha_{2}$ were gradually decreased starting from $1 \cdot 10^{-3}$ in the course of the characterization procedure. Final values of the parameters $\alpha_{1}$ and $\alpha_{2}$ were equal to $1 \cdot 10^{-10}$, and obtained solutions remained stable.

Typical fittings of measured ellipsometric data are shown in Fig. 5. Refractive indices and extinction coefficients obtained in the course of the characterization process are collected in Fig. $\underline{6}$. The values of thicknesses $\delta$ obtained in the course of the characterization process are presented in the third column of Table 1 . It is obvious from Fig. 6 and Table 1 that there is a good correspondence between obtained results. Higher values of effective thicknesses corre-
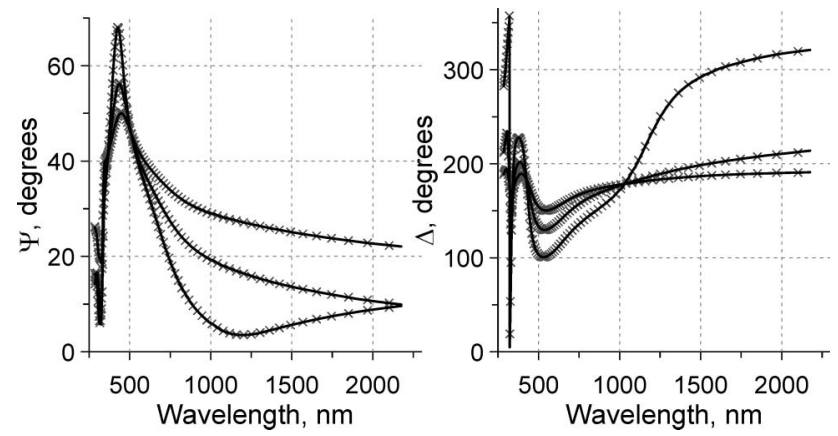

Fig. 5. Typical fittings of measurement ellipsometric data (crosses) by theoretical ellipsometric values of $\Psi$ and $\Delta$ (solid curves). The data correspond to sample S3. tion peak can be identified in the short wavelength range for all the samples, which can be attributed to interband transitions of silver [21].

At the next step of our characterization process, we verified our characterization results. We compared the theoretical reflectance and transmittance of the samples calculated based on the obtained values of $n(\lambda), k(\lambda)$, and $\delta$ with measurement $R$ and $T$ data. Typical fitting of measurement $R$ and $T$ data is shown in Fig. 7. The standard deviation between experimental and theoretical data in this case is equal to $0.7 \%$. For all other samples, the standard deviation does not exceed $2.5 \%$. These deviations can be attributed to factors related to differences in measurement devices and measurement procedures. They can be also explained by anisotropy of the MIFs. Metal clusters usually have elliptical shapes and the surface plasmon resonance depends on the light polarization, becoming sensitive to the angle of incidence. Even for a film of spherical particles, a slight anisotropy can be expected due to different particle interactions depending on light polarization
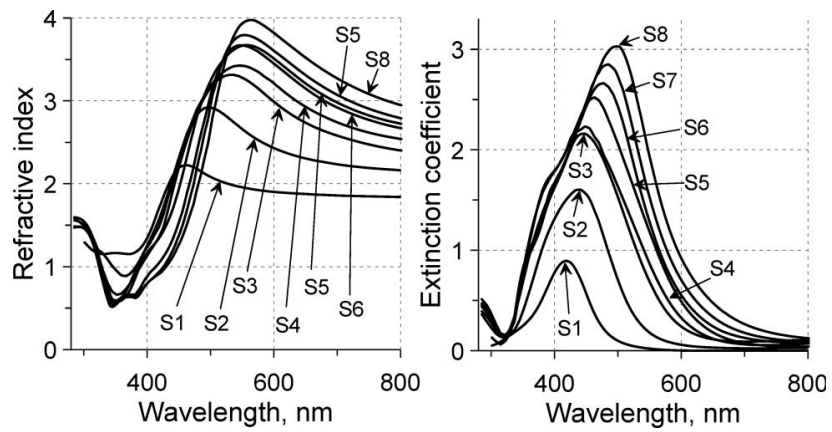

Fig. 6. Wavelength dependencies of refractive indices and extinction coefficients of $\mathrm{Ag}-\mathrm{SiO}_{2}$ composite films. The labels $\mathrm{S} 1, \ldots, \mathrm{S} 8$ indicate the corresponding samples. 

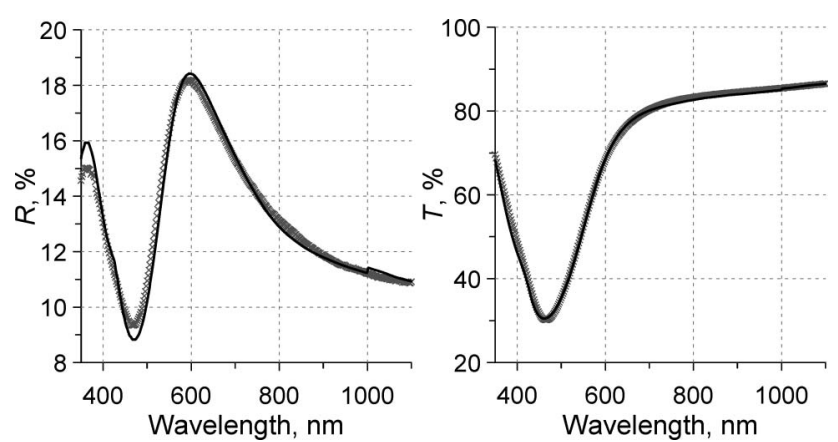

Fig. 7. Fitting of measurement spectral photometric data related to sample S3 by theoretical reflectance and transmittance calculated basing on $n$ and $k$ presented in Fig. $\underline{6}$ and $\delta=18 \mathrm{~nm}$.

$[43,44]$. On the whole, there is a good agreement of the obtained characterization results with spectral photometric measurements.

\section{B. Parametrization of Refractive Indices and Extinction Coefficients of Thin Metal Films}

To obtain analytical dependencies of refractive indices and extinction coefficients of metal films on the wavelength $\lambda$ and the thickness $\delta$, we propose a two-step procedure. At the first step, we propose to approximate the wavelength dependencies of $n$ and $k$ obtained in the course of the characterization process by simple parametric models. The key issue is that these models are to be described by as small a number of parameters as possible. At the second step, the values of these parameters are to be fitted by functions dependent on thickness $\delta$. These functions can be found empirically. Composing two obtained dependencies will provide dependencies $n(\lambda, \delta)$ and $k(\lambda, \delta)$. Below we demonstrate application of this approach to determine wavelength and thickness dependencies of $n$ and $k$ in the case of $\mathrm{Ag}-\mathrm{SiO}_{2}$ composite films.

At the first step of the procedure described above, we chose a model to approximate $n$ and $k$ values, shown in Fig. 6 in the visible spectral range. From the analysis of the optical constants obtained, it is observed that the surface plasmon resonances are characterized by an inhomogeneously broaden absorption. Such a line shape of the dispersion of the optical constants can be obtained, for instance, with the Forouhi-Bloomer model [45]:

$$
\begin{aligned}
& k(E)=\frac{A\left(E-E_{g}\right)^{2}}{E^{2}-B E+C}, \quad n(E)=n_{\infty}+\frac{B_{\alpha} E+C_{\alpha}}{E^{2}-B E+C}, \\
& B_{\alpha}=\frac{A}{Q}\left(-\frac{B^{2}}{2}+E_{g} B-E_{g}^{2}+C\right), \\
& C_{\alpha}=\frac{A}{Q}\left(\left[E_{g}^{2}+C\right] \frac{B}{2}-2 E_{g} C\right), \quad Q=\frac{1}{2} \sqrt{4 C-B^{2}}, \\
& E=1240 / \lambda, \quad E_{g}=1240 / \lambda_{g} .
\end{aligned}
$$

The Forouhi-Bloomer model was originally developed to describe electronic interband transitions in amorphous dielectrics and semiconductors, so no physical meaning should be attributed to the Forouhi-Bloomer parameters obtained in the present study. It has to be remarked that the model is only used as a mathematical tool to describe the dispersion of the optical constants of MIFs in a simple way and to verify their Kramers-Kronig consistency. The Forouhi-Bloomer model is applied in the visible range, above $400 \mathrm{~nm}$, to avoid the influence of the silver interband transitions and focus only on the surface plasmon resonance phenomenon.

From a mathematical point of view, the evident advantage of this model is that the dependence $k(\lambda)$ is described by only four model parameters, $A, B, C$, and $\lambda_{g}$. Dependence $n(\lambda)$ is described by five parameters: the same four parameters as for $k(\lambda)$ and one additional parameter, $n_{\infty}$, which specifies the shift of $n(\lambda)$ in magnitude. It is known from [45] that the preliminary values of the parameters $\bar{B}$ and $C$ can be defined from the peak of $k(\lambda)$ : value $B / 2$ is approximately equal to the energy at which $k(E)$ is a maximum; the parameter $C$ is equal to $B^{2} / 4$. The parameter $A$ gives the strength of the peak in $k(E)$ [45]. We preliminarily specified the values $A, B$, and $C$ as outlined above and calculated model parameters $A, B, C$, and $\lambda_{g}$ to refine the fit of the model dependencies $k(\lambda)$ to values found from experimental data. Then, using the found values of parameters $A, B, C$, and $\lambda_{s}$, we provided the best fit of model dependencies $n(\lambda)$ to values found from experimental data with respect to only one parameter, $n_{\infty}$. Typical fittings of $n$ and $k$ values found from experimental data by model dependencies $n(\lambda)$ and $k(\lambda)$ are presented in Fig. 8.

In Fig. 9, we collected the values of parameters $A$, $B, C, \lambda_{g}$, and $n_{\infty}$ obtained for eight $\mathrm{Ag}-\mathrm{SiO}_{2}$ composite films. These values are shown by circles. According to the second step of our two-step procedure, we approximated the dependencies of the parameters $A$, $B, C$, and $\lambda_{g}$ on $\delta$ by linear functions. The solid curves in Fig. 9 show the linear dependencies $A(\delta)=a \delta+b$, $B(\delta)=\bar{c} \delta+d, C(\delta)=e \delta+f$, and $\lambda_{g}(\delta)=g \delta+h$. The parameters $a, \ldots, h$ were found using a least-square minimization method to provide the best fit to the values of parameters $A, B, C$, and $\lambda_{g}$ found at the first step of our procedure. We approximated the dependence $n_{\infty}$ on $\delta$ by a quadratic function $n_{\infty}(\delta)=$ $a_{\infty} \delta^{2}+b_{\infty} \delta+c_{\infty}$. We do not present numerical values

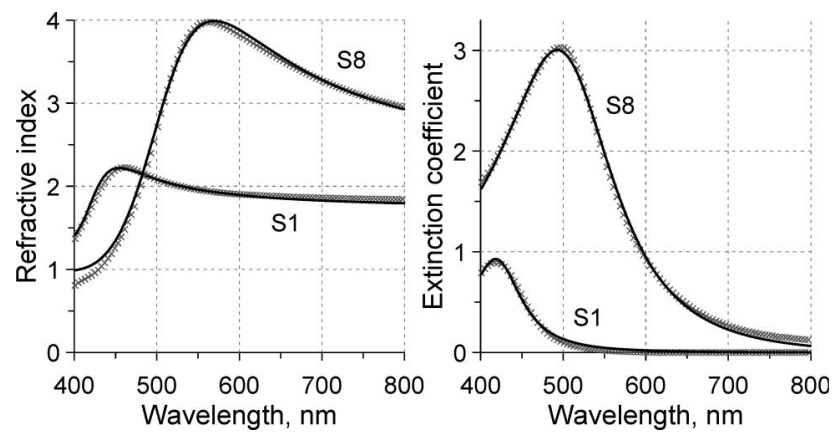

Fig. 8. Fittings of $n(\lambda)$ and $k(\lambda)$ dependencies found from experimental data by model dependencies defined by Eq. (). 

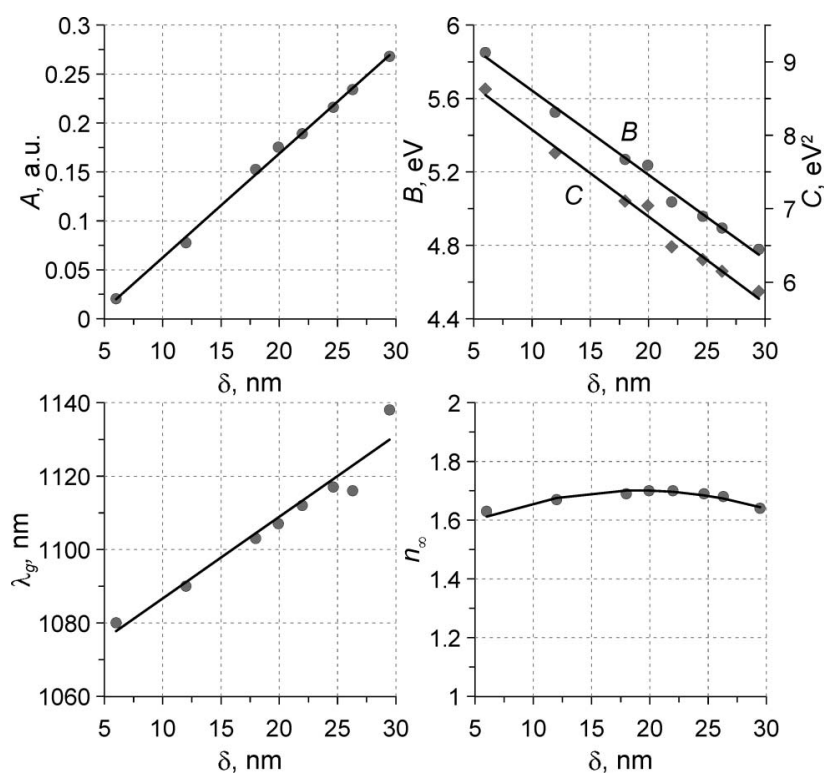

Fig. 9. Fittings of parameters $A, B, C, \lambda_{g}$ and $n_{\infty}$ obtained from experimental data (circles) by linear functions (solid curves) (see the text for details).

of the parameters $a, \ldots, h$ and $a_{\infty}, b_{\infty}$, and $c_{\infty}$ here because these values have no principal meaning.

The empirical dependencies of the refractive indices and extinction coefficients of $\mathrm{Ag}-\mathrm{SiO}_{2}$ composite films on wavelength $\lambda$ and on thickness $\delta$ can be written in the following way:

$$
\begin{aligned}
& k(\lambda, \delta)=\frac{A(\delta)\left[\lambda_{g}(\delta)-\lambda\right]^{2}}{C(\delta) \lambda^{2}-B(\delta) G \lambda+G^{2}}, \\
& n(\lambda, \delta)=n_{\infty}(\delta)+\frac{C_{\alpha}(\delta) \lambda^{2}+B_{\alpha}(\delta) G \lambda}{C(\delta) \lambda^{2}-B(\delta) G \lambda+G^{2}}, \\
& B_{\alpha}=\frac{A(\delta)}{Q(\delta)}\left\{-\frac{B^{2}(\delta)}{2}+\frac{G}{\lambda_{g}(\delta)} B(\delta)-\left[\frac{G}{\lambda_{g}(\delta)}\right]^{2}+C(\delta)\right\}, \\
& C_{\alpha}(\delta)=\frac{A(\delta)}{Q(\delta)}\left\{\left[\left(\frac{G}{\lambda_{g}(\delta)}\right)^{2}+C(\delta)\right] \frac{B(\delta)}{2}-\frac{2 G}{\lambda_{g}(\delta)} C(\delta)\right\}, \\
& Q(\delta)=\frac{1}{2} \sqrt{4 C(\delta)-B^{2}(\delta)}, \quad G=1240 .
\end{aligned}
$$

In Fig. 10, we compare experimentally determined refractive indices and extinction coefficients at three
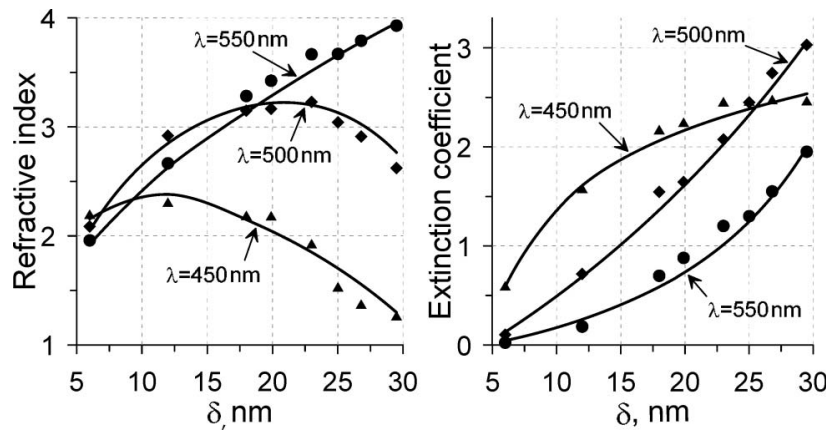

Fig. 10. Comparison of experimentally determined $n$ and $k$ values at wavelengths of 450,500 , and $550 \mathrm{~nm}$ with dependencies predicted by Eq. (9). different wavelengths with $n$ and $k$ values predicted by empirical dependencies in Eq. (9). In Fig. 10, one can observe a good correspondence of experimental results and empirical dependencies.

The dependencies of $n$ and $k$ on $\delta$ can be used in the course of elaboration of a design algorithm in the case when thin metal films are planned to be included into a coating structure.

\section{Conclusions}

In this paper, we proposed a general approach that allows us to determine reliable wavelength and thickness dependencies of the optical constants of thin metal films. In the frame of this approach, we gave recommendations what set of test samples (in particular, the range of film thicknesses) has to be produced. We have demonstrated that the choice of the proper type of measurements is critical for stable separate determination of $n, k$, and $\delta$ values. The unique and reliable solutions are obtained from ellipsometric measurements, while $R$ and $T$ measurements can be used only for verification of the characterization results. We proposed the use of nonparametric arbitrary dependencies to describe $n(\lambda)$ and $k(\lambda)$ in the course of characterization process. A two-step procedure was suggested to approximate dependencies of $n$ and $k$ on the wavelength and on the thickness of the thin metal layer. The proposed approach is universal because it allows reliable determination of optical constants as functions of wavelength and thickness of thin metal films produced with various materials and fabricated under different deposition conditions. Our approach includes a whole chain, starting from production of test samples to final determination of thin metal film optical constants $n(\lambda, \delta)$ and $k(\lambda, \delta)$. These dependencies can be incorporated into a thin film design algorithm. In this way, by optimizing the thickness of a metal film in an optical system that is being designed, the dependence of optical constants on thickness will be automatically taken into account. This approach can open new possibilities in application of thin metal films in multilayers.

\section{Appendix A}

In the frame of the first-order perturbation theory for small values of $\delta$, the spectral characteristics of symmetrical three-layer structures can be approximated as follows:

$$
\begin{aligned}
R(\lambda) & \approx R_{1}(\lambda)+\left.R^{\prime}\right|_{\delta=0}(\lambda) \delta, \\
T(\lambda) & \approx T_{1}(\lambda)+\left.T^{\prime}\right|_{\delta=0}(\lambda) \delta, \\
\Psi(\lambda) & \approx \Psi_{1}(\lambda)+\left.\Psi^{\prime}\right|_{\delta=0}(\lambda) \delta, \\
\Delta(\lambda) & \approx \Delta_{1}(\lambda)+\left.\Delta^{\prime}\right|_{\delta=0}(\lambda) \delta,
\end{aligned}
$$

where $R_{1}, T_{1}, \Psi_{1}$, and $\Delta_{1}$ are reflectance, transmittance, and the ellipsometric angles of single layers.

Calculating derivatives of $R, T, \Psi$, and $\Delta$ with respect to $\delta$ and substituting $\delta=0$ to these derivatives, 
we obtain Eqs. (2) and (7). The explicit values of $A_{R}$, $B_{R}, C_{R}, A_{T}, B_{T}$, and $C_{T}$ are as follows:

$$
\begin{aligned}
A_{R}= & 4\left[\operatorname { c o s } 2 \varphi _ { d } \left\{\left(n_{s}^{2}-1\right)\left(\cos ^{2} \varphi_{d}+\frac{n_{s}^{2}}{n_{d}^{2}} \sin ^{2} \varphi_{d}\right) \cos ^{2} 2 \varphi_{d}\right.\right. \\
& +2 n_{s}^{2} \sin ^{2} 2 \varphi_{d}\left(\cos ^{2} \varphi_{d}-\frac{\sin ^{2} \varphi_{d}}{n_{d}^{2}}\right) \\
& \left.-\left(\frac{n_{s}^{2}}{n_{d}^{2}}+n_{d}^{2}\right)\left(\cos ^{2} \varphi_{d}-\frac{n_{s}^{2}}{n_{d}^{2}} \sin ^{2} \varphi_{d}\right) \sin ^{2} 2 \varphi_{d}\right\} \\
& +\frac{1}{2} \sin ^{2} 2 \varphi_{d}\left\{\left(n_{s}^{2}-1\right)\left(1-\frac{1}{n_{d}^{2}}\right) \cos ^{2} 2 \varphi_{d}\right. \\
& \left.\left.+\left(\frac{n_{s}^{2}}{n_{d}^{2}}-n_{d}^{2}\right)\left(1-\frac{n_{s}^{2}}{n_{d}^{2}}\right) \sin ^{2} 2 \varphi_{d}\right\}\right] / D_{R T}^{2},
\end{aligned}
$$

$$
\begin{aligned}
B_{R}= & 4\left[\left(n_{d}^{2}-\frac{n_{s}^{2}}{n_{d}^{2}}\right) \frac{n_{s}}{n_{d}} \sin ^{3} 2 \varphi_{d}\right. \\
& -\frac{2 n_{s}}{n_{d}} \sin 2 \varphi_{d} \cos ^{2} 2 \varphi_{d}\left(\frac{n_{s}^{2}}{n_{d}^{2}} \sin ^{2} \varphi_{d}-n_{d}^{2} \cos ^{2} \varphi_{d}\right) \\
& +\frac{n_{s}}{2 n_{d}} \sin 4 \varphi_{d}\left(\sin ^{2} 2 \varphi_{d}\left[n_{d}^{2}+\frac{n_{s}^{2}}{n_{d}^{2}}\right]\right. \\
& \left.\left.-\left(1+n_{s}^{2}\right)\right)\right] / D_{R T}^{2}
\end{aligned}
$$$$
C_{R}=4\left[n_{d} n_{s}\left(\frac{n_{s}^{2}}{n_{d}^{2}}-n_{d}^{2}\right) \sin ^{3} 2 \varphi_{d}\right.
$$$$
+\frac{2 n_{s}}{n_{d}^{3}} \sin 2 \varphi_{d} \cos ^{2} 2 \varphi_{d}\left(\frac{n_{s}^{2}}{n_{d}^{2}} \cos ^{2} \varphi_{d}-n_{d}^{2} \sin ^{2} \varphi_{d}\right)
$$$$
+\frac{1}{2} n_{d} n_{s} \sin 4 \varphi_{d}\left(\sin ^{2} 2 \varphi_{d}\left[n_{d}^{2}+\frac{n_{s}^{2}}{n_{d}^{2}}\right]\right.
$$$$
\left.\left.-\left(1+n_{s}^{2}\right)\right)\right] / D_{R T}^{2}
$$

$$
\begin{aligned}
A_{T}= & 4\left[\operatorname { c o s } 2 \varphi _ { d } \left\{\left(n_{s}+1\right) \cos ^{2} \varphi_{d}\right.\right. \\
& \left.-\frac{1}{n_{d}^{2}}\left(1+n_{s}^{2}\right) \sin ^{2} \varphi_{d}\right\} \\
& \left.+\frac{1}{2}\left(n_{s}+\frac{n_{s}}{n_{d}^{2}}+1+\frac{n_{s}^{2}}{n_{d}^{2}}\right) \sin ^{2} 2 \varphi_{d}\right] / D_{R T}^{2} \\
B_{T}= & -4 \frac{\sin 2 \varphi_{d}}{n_{d}}\left\{0.5\left(n_{s}^{2}+1\right) \cos 2 \varphi_{d}\right. \\
& \left.+\frac{n_{s}^{2}}{n_{d}^{2}} \sin ^{2} \varphi_{d}-n_{d}^{2} \cos ^{2} \varphi_{d}\right\} / D_{R T}^{2}
\end{aligned}
$$

$$
\begin{gathered}
C_{T}=-4 \sin 2 \varphi_{d}\left\{0.5\left(n_{s}^{2}+1\right) n_{d} \cos 2 \varphi_{d}\right. \\
\left.+n_{s}^{2} \cos ^{2} \varphi_{d}-n_{d}^{4} \sin ^{2} \varphi_{d}\right\} / D_{R T}^{2},
\end{gathered}
$$

where $D_{R T}=\left(1+n_{s}\right)^{2} \cos ^{2} 2 \varphi_{d}+\left(n_{d}+n_{s} / n_{d}\right)^{2} \sin ^{2} 2 \varphi_{d}$ and $\varphi_{d}=2 \pi n_{d} d / \lambda$ is the phase thickness of the dielectric layer:

$$
\begin{aligned}
& A_{\Psi}=\frac{\beta A^{p}-A^{s} / \beta}{D_{\Psi}}, \quad B_{\Psi}=\frac{\beta B^{p}-B^{s} / \beta}{D_{\Psi}}, \\
& C_{\Psi}=\frac{\beta C^{p}-C^{s} / \beta}{D_{\Psi}}
\end{aligned}
$$

$$
\begin{aligned}
A^{p, s}= & \left(\left(q_{a}^{p, s}\right)^{2}-\left(q_{s}^{p, s}\right)^{2}\right)\left[\cos ^{3} 2 \varphi_{d}\left(q_{s}^{p, s} A_{a}^{p, s}-q_{a}^{p, s} A_{b}^{p, s}\right)\right. \\
& \left.+\sin ^{3} 2 \varphi_{d}\left(q_{s}^{p, s} A_{c}^{p, s}-q_{a}^{p, s} A_{d}^{p, s}\right)\right] \\
& +q_{a}^{p, s} q_{s}^{p, s} \sin 4 \varphi_{d}\left[\cos 2 \varphi_{d}\left(q_{a}^{p, s} A_{c}^{p, s}+q_{s}^{p, s} A_{d}^{p, s}\right)\right. \\
& \left.+\sin 2 \varphi_{d}\left(q_{a}^{p, s} A_{a}^{p, s}+q_{s}^{p, s} A_{b}^{p, s}\right)\right]-\left(\left(q_{a}^{p}\right)^{2}+\left(q_{s}^{p}\right)^{2}\right) \\
& \times \frac{1}{2} \sin 4 \varphi_{d}\left[\cos 2 \varphi_{d}\left(q_{s}^{p, s} A_{c}^{p, s}+q_{a}^{p, s} A_{d}^{p, s}\right)\right. \\
& \left.+\sin 2 \varphi_{d}\left(q_{s}^{p, s} A_{a}^{p, s}+q_{a}^{p, s} A_{b}^{p, s}\right)\right],
\end{aligned}
$$

$$
\begin{aligned}
B^{p, s}= & \left(\left(q_{a}^{p, s}\right)^{2}-\left(q_{s}^{p, s}\right)^{2}\right)\left[\cos ^{3} 2 \varphi_{d}\left(q_{s}^{p, s} B_{a}^{p, s}-q_{a}^{p, s} B_{b}^{p, s}\right)\right. \\
& \left.+\sin ^{3} 2 \varphi_{d}\left(q_{s}^{p, s} B_{c}^{p, s}-q_{a}^{p, s} B_{d}^{p, s}\right)\right] \\
& +q_{a}^{p, s} q_{s}^{p, s} \sin 4 \varphi_{d}\left[\cos 2 \varphi_{d}\left(q_{a}^{p, s} B_{c}^{p, s}+q_{s}^{p, s} B_{d}^{p, s}\right)\right. \\
& \left.+\sin 2 \varphi_{d}\left(q_{a}^{p, s} B_{a}^{p, s}+q_{s}^{p, s} B_{b}^{p, s}\right)\right]-\left(\left(q_{a}^{p}\right)^{2}+\left(q_{s}^{p}\right)^{2}\right) \\
& \times \frac{1}{2} \sin 4 \varphi_{d}\left[\cos 2 \varphi_{d}\left(q_{s}^{p, s} B_{c}^{p, s}+q_{a}^{p, s} B_{d}^{p, s}\right)\right. \\
& \left.+\sin 2 \varphi_{d}\left(q_{s}^{p, s} B_{a}^{p, s}+q_{a}^{p, s} B_{b}^{p, s}\right)\right],
\end{aligned}
$$

$$
\begin{aligned}
C^{p, s}= & \left(\left(q_{a}^{p, s}\right)^{2}-\left(q_{s}^{p, s}\right)^{2}\right)\left[\cos ^{3} 2 \varphi_{d}\left(q_{s}^{p, s} C_{a}^{p, s}-q_{a}^{p, s} C_{b}^{p, s}\right)\right. \\
& \left.+\sin ^{3} 2 \varphi_{d}\left(q_{s}^{p, s} C_{c}^{p, s}-q_{a}^{p, s} C_{d}^{p, s}\right)\right] \\
& +q_{a}^{p, s} q_{s}^{p, s} \sin 4 \varphi_{d}\left[\cos 2 \varphi_{d}\left(q_{a}^{p, s} C_{c}^{p, s}+q_{s}^{p, s} C_{d}^{p, s}\right)\right. \\
& \left.+\sin 2 \varphi_{d}\left(q_{a}^{p, s} C_{a}^{p, s}+q_{s}^{p, s} C_{b}^{p, s}\right)\right]-\left(\left(q_{a}^{p}\right)^{2}+\left(q_{s}^{p}\right)^{2}\right) \\
& \times \frac{1}{2} \sin 4 \varphi_{d}\left[\cos 2 \varphi_{d}\left(q_{s}^{p, s} C_{c}^{p, s}+q_{a}^{p, s} C_{d}^{p, s}\right)\right. \\
& \left.+\sin 2 \varphi_{d}\left(q_{s}^{p, s} C_{a}^{p, s}+q_{a}^{p, s} C_{b}^{p, s}\right)\right],
\end{aligned}
$$




$$
\begin{aligned}
& A_{a}^{p, s}=-q_{a}^{p, s} q_{s}^{p, s} \sin ^{2} \varphi_{d}, \quad A_{b}^{p, s}=\cos ^{2} \varphi_{d}, \\
& A_{c}^{p, s}=q_{a}^{p, s} \sin 2 \varphi_{d} / q_{s}^{p, s}, \quad A_{d}^{p, s}=\sin 2 \varphi_{d} / 2, \\
& B_{a}^{p, s}=-0.5 q_{a}^{p, s} q_{s}^{p, s} \sin 2 \varphi_{d}, \quad B_{b}^{p, s}=-0.5 \sin 2 \varphi_{d}, \\
& B_{c}^{p, s}=-q_{a}^{p, s} \sin ^{2} \varphi_{d} / q_{s}^{p, s}, \quad B_{d}^{p, s}=\cos ^{2} \varphi_{d}, \\
& C_{a}^{p}=-0.5 q_{a}^{p} q_{s}^{p} \sin 2 \varphi_{d}, \quad C_{b}^{p}=-0.5\left(q_{s}^{p}\right)^{2} \sin 2 \varphi_{d}, \\
& C_{c}^{p}=q_{a}^{p} q_{s}^{p} \cos ^{2} \varphi_{d}, \quad C_{d}^{p}=-\left(q_{s}^{p}\right)^{2} \sin ^{2} \varphi_{d}, \\
& C_{a}^{s}=-0.5 q_{a}^{s}\left(q_{s}^{s}-\alpha^{2} / q_{s}^{s}\right) \sin 2 \varphi_{d}, \\
& C_{b}^{s}=0.5\left(\alpha^{2}-\left(q_{s}^{s}\right)^{2}\right) \sin 2 \varphi_{d}, \\
& C_{c}^{s}=q_{a}^{s} q_{s}^{s}\left(\cos ^{2} \varphi_{d}+\alpha^{2} \sin ^{2} \varphi_{d} /\left(q_{s}^{s}\right)^{2}\right), \\
& C_{d}^{s}=-\left(\left(q_{s}^{s}\right)^{2} \sin ^{2} \varphi_{d}+\alpha^{2} \cos ^{2} \varphi_{d}\right),
\end{aligned}
$$

$$
\begin{aligned}
& A_{\Delta}=0.25 \sin 4 \varphi_{d}\left(\mu_{-}^{p} \alpha_{-}^{p} / q_{d}^{p}-\mu_{-}^{s} \alpha_{-}^{s} / q_{d}^{s}+\left(\mu_{+}^{s}\right)^{2} \alpha_{+}^{s} / q_{d}^{s}\right. \\
& \left.\quad-\left(\mu_{+}^{p}\right)^{2} \alpha_{+}^{p} / q_{d}^{p}\right) \\
& \quad+\sin 2 \varphi_{d}\left(\gamma_{+}^{p} \beta_{-}^{p} \alpha_{-}^{p}+\gamma_{-}^{p} \beta_{+}^{p} \alpha_{+}^{p}-\gamma_{+}^{s} \beta_{-}^{s} \alpha_{-}^{s}-\gamma_{-}^{s} \beta_{+}^{s} \alpha_{+}^{s}\right), \\
& B_{\Delta}=\cos 2 \varphi_{d}\left(-\gamma_{+}^{p} \mu_{-}^{p} \alpha_{-}^{p}-\gamma_{-}^{p} \mu_{+}^{p} \alpha_{+}^{p}+\gamma_{+}^{s} \mu_{-}^{s} \alpha_{-}^{s}+\gamma_{-}^{s} \mu_{+}^{s} \alpha_{+}^{s}\right) \\
& \quad+0.5 \sin 2 \varphi_{d}\left(\beta_{-}^{p} \alpha_{-}^{p} \mu_{-}^{p} / q_{d}^{p}-\beta_{+}^{p} \alpha_{+}^{p} \mu_{+}^{p} / q_{d}^{p}\right. \\
& \left.\quad+\beta_{-}^{s} \alpha_{-}^{s} \mu_{-}^{s} / q_{d}^{s}-\beta_{+}^{s} \alpha_{+}^{s} \mu_{+}^{s} / q_{d}^{s}\right), \\
& C_{\Delta}=\cos 2 \varphi_{d}\left[\left(q_{d}^{p}\right)^{2}\left(\nu_{+}^{p} \alpha_{-}^{p} \mu_{-}^{p}-\nu_{-}^{p} \alpha_{+}^{p} \mu_{+}^{p}\right)\right. \\
& \left.\quad-\alpha_{-}^{s}\left(\alpha^{2} \gamma_{+}^{s}+\nu_{+}^{s}\left(q_{d}^{s}\right)^{2}\right) \mu_{-}^{s}-\alpha_{+}^{s}\left(\alpha^{2} \gamma_{-}^{s}-\nu_{-}^{s}\left(q_{d}^{s}\right)^{2}\right) \mu_{+}^{s}\right] \\
& \quad+0.5 \sin ^{2} 2 \varphi_{d}\left[\alpha_{-}^{p} q_{d}^{p} \mu_{-}^{p} \beta_{-}^{p}-\alpha_{+}^{p} q_{d}^{p} \beta_{+}^{p} \mu_{+}^{p}\right. \\
& \left.\quad+\left(\alpha_{-}^{s} \beta_{-}^{s} \mu_{-}^{s}-\alpha_{+}^{s} \beta_{+} \mu_{+}^{s}\right)\left(\alpha^{2} / q_{d}^{s}-q_{d}^{s}\right)\right],
\end{aligned}
$$

$$
\begin{aligned}
& \mu_{+,-}^{s, p}=q_{a}^{s, p}-q_{s}^{s, p}, \quad \beta_{+,-}^{s, p}=q_{a}^{s, p} q_{s}^{s, p} / q_{d}^{s, p} \pm q_{d}^{s, p} \\
& \gamma_{+,-}^{s, p}=\cos ^{2} \varphi_{d} \pm q_{a}^{s, p} q_{s}^{s, p} \sin ^{2} \varphi_{d} /\left(q_{d}^{s, p}\right)^{2} \\
& \nu_{ \pm}^{s, p}=q_{a}^{s, p} q_{s}^{s, p} \cos ^{2} \varphi_{d} /\left(q_{d}^{s, p}\right)^{2} \pm \sin ^{2} \varphi_{d} \\
& \alpha_{+,-}^{s, p}=\left[\left(\mu_{+,-}^{s, p}\right)^{2} \cos ^{2} 2 \varphi_{d}+\left(\beta_{+,-}^{s, p}\right)^{2} \sin ^{2} 2 \varphi_{d}\right]^{-1} \\
& q_{a, s, d}^{s}=\sqrt{\left(n_{a, s, d}\right)^{2}-\alpha^{2}} \\
& q_{a, s, d}^{p}=\left(n_{a, s, d}\right)^{2} / \sqrt{\left(n_{a, s, d}\right)^{2}-\alpha^{2}}, \quad \alpha=n_{a} \sin ^{2} \theta .
\end{aligned}
$$

Equation (A14) was obtained assuming $\alpha^{2} /\left[(\operatorname{Re} \epsilon)^{2}+(\overline{\operatorname{Im} \epsilon})^{2}\right]<1$.

The authors of the paper are grateful to the National Foundation of Science, Higher Education and Technological Development of the Republic of Croatia, which has partially funded the research.

\section{References}

1. N. Kaiser, "Review of the fundamentals of thin-film growth," Appl. Opt. 41, 3053-3060 (2002).

2. N. Kaiser, "Optical coatings road-map," presented at the International Workshop on Optical Coatings In Celebration of Dr. J. A. Dobrowolski's 50th Year at NRC, Ottawa, Canada, 11 May 2006.

3. P. Heger, O. Stenzel, and N. Kaiser, "Design and fabrication of selective thin film absorbers on the basis of silver island films," Vakuum in Forschung und Praxis 18, 53-56 (2006).

4. A. Stepanov, "Optical transmission of dielectric layers with metallic nanoparticles inhomogeneously distributed over the sample thickness," Opt. Spectrosc. 91, 815-819 (2001).

5. A. Lehmuskero, M. Kuittinen, and P. Vahimaa, "Refractive index and extinction coefficient dependence of thin al and ir films on deposition technique and thickness," Opt. Express 15, 10744-10752 (2007).

6. O. Stenzel, A. Stendal, M. Röder, S. Wilbrandt, D. Drews, T. Werninghaus, C. von Borczyskowski, and D. Zahn, "Localized plasmon excitation in metal nanoclusters as a tool to study thickness-dependent optical properties of copper phthalocyanine ultrathin films," Nanotechnology 9, 6-19 (1998).

7. O. Stenzel, A. Stendal, M. Röder, and C. von Borczyskowski, "Tuning of the plasmon absorption frequency of silver and indium nanoclusters via thin amorphous silicon films," Pure Appl. Opt. 6, 577-588 (1997).

8. A. Leitner, Z. Zhao, H. Brunner, F. Aussenegg, and A. Wokaun, "Optical properties of a metal island film close to a smooth metal surface," Appl. Opt. 32, 102-110 (1993).

9. I. Hooper and J. Sambles, "Some considerations on the transmissivity of thin metal films," Opt. Express 16, 17249-17257 (2008).

10. Y. Jourlin, S. Tonchev, A. Tishchenko, C. Pedri, C. Veillas, O. Parriaux, A. Last, and Y. Lacroute, "Spatially and polarization resolved plasmon mediated transmission through continuous metal films," Opt. Express 17, 12155-12166 (2009).

11. T. Iwata and G. Komoda, "Measurements of complex refractive indices of metals at several wavelengths by frustrated total internal reflection due to surface plasmon resonance," Appl. Opt. 47, 2386-2391 (2008).

12. M. Hövel, B. Gompf, and M. Dressel, "Dielectric properties of ultrathin metal films around the percolation threshold," Phys. Rev. B 81, 035402 (2010).

13. A. Nabok, A. Tsargorodskaya, and Suryajaya, "Ellipsometry study of ultra thin layers of evaporated gold," Phys. Status Solidi C 5, 1150-1155 (2008).

14. A. J. de Vries, E. S. Kooij, H. Wormeester, A. A. Mewe, and B. Poelsema, "Ellipsometric study of percolation in electroless deposited silver films," J. Appl. Phys. 101, 053703 (2007).

15. W. Chen, M. D. Thoreson, S. Ishii, A. V. Kildishev, and V. M. Shalaev, "Ultra-thin ultra-smooth and low-loss silver films on a germanium wetting layer," Opt. Express 18, 5124-5134 (2010).

16. H. Zorc, M. Lončarić, J. Sancho-Parramon, and V. Janicki, "Use of gold island films in design of reflectors with high luminosity," in Optical Interference Coatings (Optical Society of America, 2010), paper TuD8.

17. J. A. Dobrowolski, S. Browning, M. Jacobson, and M. Nadal, "2007 Topical Meeting on Optical Interference Coatings: manufacturing problem," Appl. Opt. 47, C231-C245 (2008).

18. J. A. Dobrowolski, S. Browning, M. R. Jacobson, and M. Nadal, "2004 Optical Society of America's Topical Meeting on Optical Interference Coatings: manufacturing problem," Appl. Opt. 45, 1303-1311 (2006).

19. E. D. Palik, Handbook of Optical Constants of Solids (Academic, 1985).

20. N\&K database, http://www.sopra-sa.com/.

21. P. B. Johnson and R. W. Christy, "Optical constants of the noble metals," Phys. Rev. B 6, 4370-4379 (1972).

22. H. Liu, B. Wang, E. S. P. Leong, P. Yang, Y. Zong, G. Si, J. Teng, and S. A. Maier, "Enhanced surface plasmon resonance on a 
smooth silver film with a seed growth layer," ACS Nano 4, 3139-3146 (2010).

23. U. Kreibig and M. Vollmer, Optical Properties of Metal Clusters (Springer, 1995).

24. J. A. Dobrowolski, L. Li, and R. A. Kemp, "Metal/dielectric transmission interference filters with low reflectance. 1. Design,” Appl. Opt. 34, 5673-5683 (1995).

25. P. Ma, F. Lin, and G. J. Dobrowolski, "Design and manufacture of metal-dielectric long wavelength cut-off filters," in Optical Interference Coatings (Optical Society of America, 2010), paper MA9.

26. A. V. Tikhonravov, M. K. Trubetskov, O. F. Prosovskiy, and M. A. Kokarev, "Optical characterization of thin metal films," in Optical Interference Coatings (Optical Society of America, 2007), paper WDPDP2.

27. A. Tikhonravov, M. Trubetskov, T. Amotchkina, M. Kokarev, I. Kozlov, V. Zhupanov, E. Kluev, and O. Prosovskiy, "Optical coatings containing well-controlled few nanometer thick metal layers," in Nanofair 2008. New Ideas for Industry (WDI Wissensforum GmbH, 2008), pp. 171-174.

28. A. B. Djurišić, T. Fritz, and K. Leo, "Determination of optical constants of thin absorbing films from normal incidence reflectance and transmittance measurements," Opt. Commun. 166, 35-42 (1999).

29. M. Lončarić, J. Sancho-Parramon, M. Pavlović, H. Zorc, P. Dubček, A. Turković, S. Bernstorff, G. Jakopic, and A. Haase, "Optical and structural characterization of silver islands films on glass substrates," Vacuum 84, 188-192 (2009).

30. J. Sancho-Parramon, V. Janicki, J. Arbiol, H. Zorc, and F. Peiro, "Electric field assisted dissolution of metal clusters in metal island films for photonic heterostructures," Appl. Phys. Lett. 92, 163108 (2008).

31. V. Janicki, J. Sancho-Parramon, F. Peiro, and J. Arbiol, "Threedimensional photonic microstructures produced by electric field assisted dissolution of metal nanoclusters in multilayer stacks," Appl. Phys. B 98, 93-98, doi: 10.1007/s00340-0093705-7 (2010).

32. A. N. Tikhonov and V. Y. Arsenin, Solution of Ill-Posed Problems (Winston-Wiley, 1977).

33. J. Sancho-Parramon, J. Ferré-Borrull, S. Bosch, and M. C. Ferrara, "Use of information on the manufacture of samples for the optical characterization of multilayers through a global optimization," Appl. Opt. 42, 1325-1329 (2003).
34. A. Lebedev, O. Stenzel, M. Quinten, A. Stendal, M. Röder, M. Schreiber, and D. Zahn, "A statistical approach for interpreting the optical spectra of metal island films: effects of multiple scattering in a statistical assembly of spheres," J. Opt. A Pure Appl. Opt. 1, 573-580 (1999).

35. W. Vargas, D. Azofeifa, N. Clark, and X. Márquez, "Collective response of silver islands on a dielectric substrate when normally illuminated with electromagnetic radiation," J. Phys. D 41, 025309 (2008).

36. O. Stenzel, The Physics of Thin Film Optical Spectra (Springer-Verlag, 2005).

37. A. V. Tikhonravov, M. K. Trubetskov, J. Hrdina, and J. Sobota, "Characterization of quasi-rugate filters using ellipsometric measurements," Thin Solid Films 277, 83-89 (1996).

38. O. Stenzel and R. Petrich, "Flexible construction of error functions and their minimization: application to the calculation of optical constants of absorbing or scattering thin-film materials from spectrophotometric data," J. Phys. D 28, 978-989 (1995).

39. A. Tikhonravov and M. Trubetskov, "Optical characterization of thin films-basic concept,", presented at the XIV OptiLayer Workshop in Europe, Hannover, 22-24 March 2010, and the VII OptiLayer Workshop in the USA, Santa Clara, 16-18 November 2009.

40. A. Tikhonravov and M. Trubetskov, "Thin film characterization using spectral ellipsometric data," presented at the XIV OptiLayer Workshop in Europe, Hannover, 22-24 March 2010, and the VII OptiLayer Workshop in the USA, Santa Clara, 16-18 November 2009.

41. A. V. Tikhonravov and M. K. Trubetskov, OptiLayer thin film software, http://www.optilayer.com.

42. J. Sancho-Parramon, "Surface plasmon resonance broadening of metallic particles in the quasi-static approximation: a numerical study of size confinement and interparticle interaction effects," Nanotechnology 20, 235706 (2009).

43. R. R. Singer, A. Leitner, and F. R. Aussenegg, "Structure analysis and models for optical constants of discontinuous metallic silver films," J. Opt. Soc. Am. B 12, 220-228 (1995).

44. R. G. Barrera, M. del Castillo-Mussot, G. Monsivais, P. Villaseor, and W. L. Mochán, "Optical properties of twodimensional disordered systems on a substrate," Phys. Rev. B 43, 13819-13826 (1991).

45. A. Forouhi and I. Bloomer, "Calculation of optical constants, $n$ and $k$, in the interband region," in Handbook of Optical Constants of Solids II, E. Palik, ed. (Academic, 1991), pp. 151-175. 